\title{
COMPARACION FLORISTICA DE DOCE PARCELAS EN BOSQUE DE TIERRA FIRME EN LA AMAZONIA PERUANA.
}

\author{
Kalle RUOKOLAINEN', Hanna TUOMISTO', Richer RÍOS², Alberto TORRES', \\ Mildred GARCÍA ${ }^{3}$
}

ABSTRACT - Floristic composition of twelve plots, $25 \mathrm{~m}$ by $25 \mathrm{~m}$, was studied in the Peruvian Amazonia in order to investigate if it were possible to use a part of the flora as an indicator of the changes observed in other components of the flora. Floristic similarities among the plots were calculated using six different taxonomic or physiognomic groups: ferns, the families Melastomataceae and Myristicaceae, trees thicker than $2.5 \mathrm{~cm}$ diameter at breast hight (DBH), trees between $2.5 \mathrm{~cm}$ and $5 \mathrm{~cm} \mathrm{DBH}$, and trees thicker than $5 \mathrm{~cm} \mathrm{DBH}$. The similarities were used for agglomerative classifications of the plots and for analyzing the correlations among the different groups of plants with Mantel's test. The results indicate that floristic changes were similar in all of the groups and therefore every group can potentially be used as an indicator.

Key words: Amazonia, ferns, Mantel's test, Melastomataceae, Myristicaceae, phytosociology.

Comparação Florística de Doze Parcelas Numa Floresta de Terra Firme da Amazônia Peruana.

RESUMO - Um estudo da composição florística de doze parcelas de $25 \mathrm{~m} \times 25 \mathrm{~m}$ foi realizado na Amazônia peruana com o objetivo de observar a possibilidade de utilizar uma parte da flora como indicador para as alterações anotadas em outros elementos da flora. As similaridades florísticas entre as parcelas foram definidas usando seis grupos taxonômicos ou fisionômicos diferentes: fetos, as familias Melastomataceae e Myristicaceae, árvores com diâmetro á altura do peito (DAP) maior a $2.5 \mathrm{~cm}$, árvores com DAP entre $2.5 \mathrm{~cm}$ e $5 \mathrm{~cm}$, e árvores maiores a $5 \mathrm{~cm}$ de DAP. As similaridades foram utilizadas para classificar as parcelas e para analisar as correlações entre os grupos diferentes de plantas com a prova de Mantel. Os resultados mostram que as alterações florísticas são similares em todos os grupos, e por isso qualquer destes grupos é capaz de servir de indicador.

Palavras-chave: Amazônia, fetos, fitossociologia, Melastomataceae, Myristicaceae, prova de Mantel.

\section{INTRODUCCION}

La información sobre la distribución de especies de plantas y sobre las diferencias y semejanzas florísticas entre distintos lugares en la Amazonía juegan un papel importante tanto en los estudios científicos de la biota amazónica como en la planificación del manejo y conservación de esta naturaleza. Por ejemplo, los citados centros del endemismo de plantas en la Amazonía (PRANCE, 1973, 1982) han formado una parte importante en la teoría de los refugios pleistocénicos (HAFFER, 1969) y en la identificación de las áreas de mayor importancia en la conservación de la biota amazónica (CONSERVATION INTERNATIONAL, 1991). Sin embargo, el conocimiento actual sobre distribución de las especies vegetales en la Amazonía es inadecuado para definir centros de endemismo y patrones fitogeográficos. Las colecciones botánicas en la región son pequeñas, y además están concentradas en pocas localidades (CAMPBELL \& HAMMOND, 1989; NELSON et al., 1990).

1 Department of Biology, University of Turku, SF-20500 Turku, Finlandia.

2 Facultad de Ingenieria Forestal, Universidade Nacional de la Amazonia Peruana, Iquitos, Peru.

3 Facultad de Ciencias Biológicas, Universidad Nacional de la Amazonia Peruana, Iquitos, Peru. 
Para investigar los patrones fitogeográficos sería mejor utilizar la información cuantitativa de inventarios botánicos sistemáticos en áreas seleccionadas, que utilizar colecciones botánicas generales. No obstante, los inventarios botánicos en la Amazonía son escasos (CAMPBELL et al. 1986; SALOMÃO et al. 1988, y referencias en los mismos) y todavía no permiten evaluar las relaciones florísticas exactas de las diferentes partes de la Amazonía. Lo que se puede decir es que existe una gran variación en la composición florística entre los diferentes sitios, sean estos lejanos o cercanos entre si. Estas diferencias florísticas entre parcelas pueden tener por lo menos dos razones. Una es fitogeográfica, la otra es ecológica. Sobre suelos diferentes crecen especies diferentes, y en consecuencia la composición florística de la vegetación puede variar bastante en distancias muy cortas, hasta en la selva amazonica que superficialmente parece homogénea (RUOKOLAINEN \& TUOMISTO, 1993; TUOMISTO et al., en prensa; TUOMISTO \& RUOKOLAINEN, en prensa). Por lo tanto, para justificar conclusiones fitogeográficos es necesario conocer en que tipo de vegetación se han realizado los estudios del campo, y como es la distribución de los diferentes tipos de vegetación.

Las clasificaciones de la vegetación que más se utilizan en las áreas templadas del mundo, se basan en la composición floristica de la vegetación. Tales clasificaciones pueden definir los tipos de vegetación utilizando toda la flora presente (BRAUN-BLANQUET, 1932), las especies dominantes (WHITTAKER,
1962), o algunas especies preseleccionadas por su valor indicativo (CAJANDER, 1909). El problema en aplicar estos métodos en los bosques húmedos tropicales, además de varios problemas logísticos, ha sido la gran riqueza de especies en los bosques. En los estudios de campo es imposible conocer todas las especies presentes, y hacer colecciones botánicas de casi todos los individuos de plantas resulta muy laborioso. El uso de las especies dominantes generalmente no es mucho más fácil, porque la gran diversidad florística de los bosques hace que antes de haber identificado todos los individuos no se puede saber cuales son los dominantes, sí es que existen especies dominantes. Por otra parte, el estudio de las especies indicadoras no ha sido posible debido a que se desconoce la ecología de las especies de plantas, y en consecuencia no hay criterios para elegir los indicadores.

Una alternativa para evitar por lo menos una parte de los problemas de la gran diversidad de especies es concentrar el muestreo en individuos de un grupo taxonómico, forma de vida y/o tamaño definido. Evidentemente, mucho más fácil que estudiar toda la flora es estudiar por ejemplo solamente los helechos, y establecer los tipos de vegetación y los patrones fitogeográficos según las distribuciones de diferentes especies de helechos. Este enfoque se basa en la idea que existen verdaderas comunidades de especies o asociaciones de ciertas especies que casi siempre aparecen juntas. De esta manera la presencia de algunas especies significa que en el mismo lugar existen con alta probabilidad también las otras especies de la asociación. 
La presencia de comunidades identificables en las zonas termpladas es bien establecida. Pero, sobre los bosques pluviales tropicales existen visiones que varían desde una composición aleatoria de especies (HUBBELL \& FOSTER, 1986) hasta la presencia de especies estrictamente controlada por factores ambientales (ASHTON, 1969; GENTRY, 1981; 1988).

En los estudios fitosociológicos de los bosques húmedos tropicales ha sido común estudiar solamente los árboles que tienen diámetro a la altura del pecho (DAP) mayor que un limite pre-seleccionado, frecuentemente 10 $\mathrm{cm}$. Este costumbre aparentemente ha nacido de la tradición forestal de sólo incluir árboles grandes en los inventarios. El uso de árboles grandes en los estudios fitosociológicos es soportado por los resultados de WEBB et al. (1967), quienes han evaluado las posibilidades de usar diferentes formas de vida en la clasificación de bosques húmedos tropicales en Australia. Lamentablemente el uso de árboles mayores de $10 \mathrm{~cm}$ DAP presenta algunos problemas prácticos: el muestreo de los individuos es muy laborioso, y para obtener suficientes individuos en una muestra representativa, hay que realizar parcelas tan grandes que incluyen bastante variación topográfica y edáfica.

En la práctica, resulta más conveniente incluir en el estudio solamente plantas pequeñas y fáciles de colectar, o grupos taxonómicos que pueden reconocerse en el campo. Con esos criterios se eligieron los helechos, las Melastomatấceas y las Myristicáceas para el presente estudio. Estos grupos son abundantes en los bosques amazónicos y especialmente los helechos y las Melastomatáceas presentan muchas especies incluyendo representantes de diferentes formas de vida, tales como hierbas, trepadoras, arbustos y árboles. Igualmente, se estudiaron árboles mayores de $2.5 \mathrm{~cm}$ DAP ("todos los árboles"), los cuales fueron divididos para los análisis en dos grupos, árboles entre $2.5 \mathrm{~cm}$ y $5 \mathrm{~cm}$ DAP ("árboles pequeños") y árboles mayores de $5 \mathrm{~cm}$ DAP ("árboles grandes").

El objetivo del presente estudio es comparar los diferentes grupos de plantas en cada parcela, y determinar sí existe una buena correlación entre los grupos, que justifique así el uso de uno o varios grupos como indicadores de la distribución de otros grupos de especies.

\section{Área del Estudio}

El estudio se realizó en dos localidades de la Amazonía peruana en las cercanías a la ciudad Iquitos. EI primer lugar se encuentra en el terreno de la empresa turística, Amazon Selva Tours, ubicado aproximadamente 15 $\mathrm{km}$ al noroeste de Iquitos en el Río Momón (73' 36' W, 03" 36' S; aprox. 120 m s.n.m.). El segundo lugar está ubicado cerca del pueblo de Mishana en el Río Nanay, aproximadamente 30 $\mathrm{km}$ al suroeste de Iquitos (73' $32^{\prime} \mathrm{W}$, 3" 51' S; aprox. 150 m s.n.m.; Fig. 1). El clima de la zona es húmedo tropical, y se caracteriza por lluvias abundantes casi todo el año. La precipitación media anual es aproximadamente $2600 \mathrm{~mm}$, y las temperaturas mínimas y máximas alcanzan $15,0^{\circ} \mathrm{C}$ y $35,5^{\circ} \mathrm{C}$, respectivamente, con un promedio anual de $25,9^{\circ} \mathrm{C}$ (PEÑA HERRERA. 1986). 


\section{Río Momón}

Los alrededores del Río Momón se encuentran bajo cultivo intensivo, y el área estudiada está situada en un bosque rodeado por un mosaico de parcelas cultivadas. El lugar ha permanecido prácticamente intacto desde el establecimiento del albergue turístico en los años 1980. Sin embargo, es probable que el bosque haya sido afectado por la tala de árboles en décadas anteriores.

Toda la zona estudiada corresponde a tierra firme (no inundable por la creciente del río) con una topografía generalmente plana. Existe una leve ondulación con colinas hasta $10 \mathrm{~m}$ de altura cuando se aleja del río, probablemente indicando la existencia de una antigua terraza del río Momón. Los suelos de la zona son arcillosos. En las depresiones se encuentra una vegetación pantanosa dominada por la palmera Mauritia

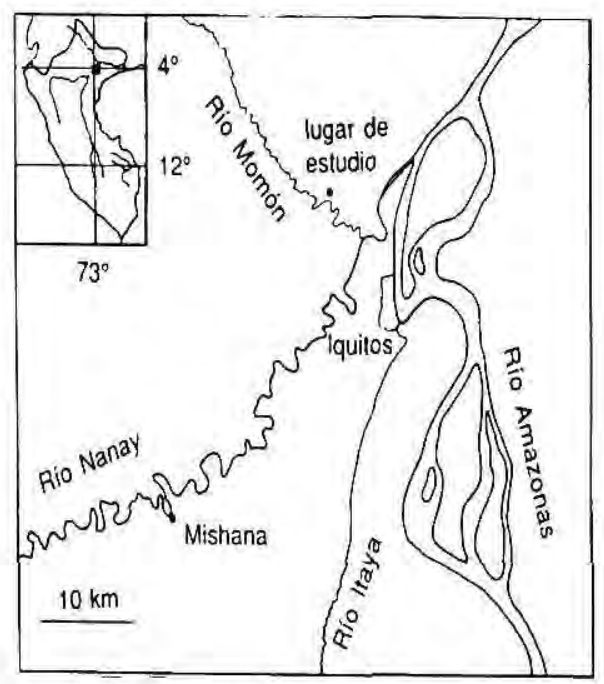

Figura 1. Ubicación de las áreas del muestro. flexuosa L.f. (aguaje). Estas áreas fueron excluidas del presente estudio.

\section{Mishana}

Después de la zona inundable por el Río Nanay, hacia el sur, existe una zona de tierra firme de terreno plano y con suelos areno-arcillosos mal drenados. En algunas partes se encuentran pozos poco profundos que colectan agua después de las lluvias secandose en pocos días; en estos lugares la mayoría de las plantas crece entre los pozos donde el drenaje es mejor. Las palmeras son comunes entre los árboles, el sotobosque es denso y está dominado por plantas con hojas grandes. Hacia el sur, el terreno presenta colinas de aproximadamente 15 metros sobre el nivel de las quebradas, las cuales están disectadas casi hasta el nivel de la terraza. En las lomas de las colinas el suelo es arenoso, observandose pura arena blanca de quarzo. La vegetación sobre arenas blancas se denomina a varillal (ENCARNACIÓN, 1985) y se caracteriza por abundantes árboles delgados con hojas esclerófilas, y escasos arbustos e hierbas en el sotobosque. Los helechos pequeños son abundantes, y en algunos partes se encuentran Bromeliaceaes creciendo en el suelo.

\section{MUESTREO Y ANALISIS DE LOS DATOS}

Se establecieron en total 12 parcelas de $25 \mathrm{~m}$ por $25 \mathrm{~m}$ ( 0.0625 ha). En Momón se ubicaron cuatro parcelas, dos (parcelas 1 y 2) en la parte baja y plana, y otras dos (3 y 4 ) en la zona más alta y colinosa. En las últimas parcelas se observaron pe- 
queños claros por causa de árboles caídos. En Mishana se ubicaron ocho parcelas en fila, cuatro parcelas ( 5 a 8 ) se establecieron en la terraza baja, y cuatro ( 9 a 12) en el terreno colinoso de arena blanca. Las parcelas 5,6 y 8 presentaron mal drenaje, en la parcela 7 un drenaje relativamente bueno, y las parcelas 9 a 12 un drenaje excesivo. La parcela 8 estaba situada en la zona de transición entre las dos unidades fisiográficas, las parcelas 9 y 10 estaban en las lomas de la primera colina y parcelas 11 y 12 en las lomas de la segunda colina del terreno ondulado.

Los sitios para las parcelas fueron elegidos de tal-manera que parecían representativos de los alrededores y no demasiado heterogéneos. Especialmente se trató de evitar claros recientes. Todas las parcelas fueron divididas en subparcelas de $5 \mathrm{~m}$ por $5 \mathrm{~m}$ y los datos se colectaron en cada subparcela. En los árboles mayores a $2.5 \mathrm{~cm}$ DAP se midieron las circunferencias, y se estimó la altura de las Melastomataceas. De los helechos se elaboró una lista de especies para cada parcela. Para la identificación de las especies, se colectaron todas las plantas que no podían referirse a alguna especie ya colectada.

Los duplicados de las muestras botánicas se encuentran depositados en el Herbario de la Universidad de Turku (TUR), en el Herbarium Amazonense (AMAZ), y en el Museo de Historia Natural "Javier Prado" de la Universidad Nacional Mayor de San Marcos (USM). La identificación de las muestras se realizó principalmente por comparación con muestras depositados en el Missouri Botanical Garden; las colecciones en AMAZ, USM y los herbarios de las universidades de Utrecht y Aarhus fueron también consultados. Varios grupos de plantas fueron identificados por taxónomos especialistas.

Las relaciones fitosociológicas entre las parcelas fueron analizados con una clasificación aglomerativa y con la prueba de Mantel. Los análisis se basaron en las similitudes florísticas calculadas entre todos los pares posibles de parcelas, usando individualmente cada uno de los grupos de plantas incluidos en el estudio. Las similitudes fueron expresadas a través del índice de JACARRD (1901) en el caso de los helechos, y a través del porcentaje de similitud de BRAY \& CURTIS (1957) para todos los otros grupos de plantas.

Los índices de similitud pueden tener valores entre 0 y 1 , cero indicando que ninguna especie crece en ambas parcelas comparadas, y uno indicando una igualidad florística total. El valor del índice de Jaccard (J) entre dos parcelas está determinado por la ausencia o presencia de las especies, y se calcula según la siguiente fórmula:

$$
\mathrm{J}=\mathrm{c} /(\mathrm{a}+\mathrm{b}+\mathrm{c})
$$

donde c es el número de especies encontradas en ambas parcelas, a es el número de especies encontradas en sólo una de las parcelas, y b es el número de especies encontradas sólo en la otra parcela.

El porcentaje de similitud considera las abundancias relativas de las especies, y se calcula según la siguiente fórmula:

$\mathrm{PS}_{\mathrm{ij}}=2 \mathrm{~S}_{\mathrm{k}} \min \left(\mathrm{x}_{\mathrm{ki}}, \mathrm{x}_{\mathrm{kj}}\right) /\left(\mathrm{S}_{\mathrm{k}} \mathrm{x}_{\mathrm{ki}}+\mathrm{S}_{\mathrm{k}} \mathrm{x}_{\mathrm{kj}}\right)$ donde:

$x_{k 1}$ es la abundancia de la especie $k$ en parcela $\mathrm{i}$

$\mathrm{x}_{\mathrm{kj}}$ es la abundancia de la especie $\mathrm{k}$ en 
parcela $\mathrm{j}$

$\min \left(\mathrm{x}_{\mathrm{ki}}, \mathrm{x}_{\mathrm{ki}}\right) /\left(\mathrm{S}_{\mathrm{k}} \mathrm{x}_{\mathrm{ki}}+\mathrm{S}_{\mathrm{k}} \mathrm{x}_{\mathrm{kj}}\right)$ es el valor menor de abundancia relativa que la especie $\mathrm{k}$ tiene en las dos parcelas.

Las abundancias relativas de las especies que se necesitan para calcular PSij fueron expresadas según el índice de valor de importancia (IVI; CURTIS \& McINTOSH, 1950). El IVI se utiliza comunmente en los estudios forestales y se calcula en la manera siguiente:

IVI = densidad relativa + frecuencia relativa + dominancia relativa,

donde la densidad relativa de la especie es:

$\mathrm{n}^{\circ}$ de individuos de la especie $\times \quad 100$

$\mathrm{n}^{\circ}$ total de individuos de todas las especies

la frecuencia relativa es:

$\underline{\mathrm{n}^{\circ} \text { de subparcelas ocupadas por la especie } \mathrm{x} 100}$

la suma de frecuencias de todas las especies

y la dominancia relativa es:

la suma de las áreas basales de la especie x 100 la suma de las áreas basales de todas las especies

Al calcular Ios IVIs para las Melastomataceas, se sustituyeron la área basal por la altura de los individuos.

Los valores de las similitudes se utilizaron para clasificar las parcelas con cada grupo de plantas. Las clasificaciones se realizaron según el método de los grupos de pares no balanceados (UPGMA; SOKAL \& MICHENER, 1958), donde la distancia entre dos grupos de parcelas está definida como la distancia promedio de todos los pares de parcelas posibles entre los grupos.

Para analizar si las similitudes entre las parcelas que se consiguen con un grupo de plantas son correlacionadas con las similitudes que se consiguen con otro grupo, se construyeron matrices de similitud con cada grupo de plantas y se aplicó la prueba de MANTEL (1967). Según esta, la similitud observada ( $Z$ ) entre dos matrices se calcula:

$$
\mathrm{Z}=\Sigma i \Sigma j \mathrm{x}_{i j} \mathrm{y}_{i j}
$$

donde $\mathrm{X}_{i j}$ y $\mathrm{Y}_{i j}$ corresponden a las similitudes entre las parcelas $i$ y $j \quad(i=j)$ en las dos matrices. El valor observado de $Z$ se compara con el valor esperado de $\mathrm{Z}$ que se obtiene por un método de sorteo al azar. Los valores de similitud en la matriz $X$ se mantienen fijos, mientras en la matriz Y se sortean; después se calcula $Z$ de nuevo. Cuando se repiten el sorteo y el cálculo de $Z$ varias veces, la variación en los valores de $\mathrm{Z}$ adquiere una distribución normal que permite la estimación de la probabilidad de conseguir al azar las similitudes observadas. En el presente estudio se hizo el sorteo 2000 veces. Se utilizó una forma estandardizada de $\mathrm{Z}$ (SMOUSE et al. 1986) que siempre está entre +1 (correlación positiva perfecta) y -1 (correlación negativa perfecta), y es igual a la correlación linear de Pearson. La estandardización no afecta las probabilidades asociadas. Para cumplir la condición de independencia de las matrices, los individuos de las familias Myristicaceae y Melastomatacaeae fueron excluidos de los diferentes grupos de árboles antes de comparar las matrices de similitud respectivas. En las comparaciones entre helechos y árboles, así como en las 
comparaciones entre árboles pequeños y árboles grandes, las Myristicaceas y Melastomataceas fueron incluidas en los árboles.

La clasificación aglomerativa y la prueba de Mantel se realizaron con los programas Cluster y Mantel de la compilación para análisis multivariado "R Package" por LEGENDRE \& VAUDOR (1991).

\section{RESULTADOS}

En el estudio se hallaron 2114 individuos y 517 especies de árboles mayores de $2,5 \mathrm{~cm}$, de los cuales 1111 individuos y 353 especies pertenecen a la categoria de árboles pequeños, y 1003 individuos y 377 especies a árboles grandes. Los números respectivos para Myristicaceas son 181 y 22 , y para Melastomataceas 457 y 29. Los helechos encontrados presentan 53 especies. Las especies de helechos, Melastomataceas y Myristicaceas encontradas en las diferentes parcelas se presentan en las tablas 1 a 3 , y de los árboles los valores de importancia por familia y por parcela se presentan en la Tabla 4. La lista de especies de árboles está disponible para quien la solicite del primer autor.

Las clasificaciones obtenidas según los diferentes grupos de plantas están ilustradas en las Figuras 2 a-f. Las agrupaciones según helechos y los tres grupos de árboles resultaron casi idénticas, separandose siempre cuatro grupos de parcelas ( 1 y $2 ; 3$ y $4 ; 5-7$; 9-12). Las relaciones dentro de estos grupos varían un poco. En el penúltimo grupo, las parcelas 5 y 6 siempre se encuentran cercanas entre sí. En el último grupo el orden entre las parcelas 9-12 no es fijo. La parcela 8 no presenta una posición bien definida.

Las agrupaciones según Melastomataceas y Myristicaceas difieren en relación con los patrones observados en los helechos y árboles. Las parcelas 5-7 y 9-11 permanecen agrupadas, pero las parcelas 4 y 8 se agrupan y las posiciones de las parcelas 2 y 3 cambian.

Los resultados de la prueba de Mantel se presentan en la Tabla 5. En general, los coeficientes de correlación entre los diferentes grupos de árboles y helechos son altos, y hasta las correlaciones menores son estadísticamente significativas.

\section{DISCUSION}

Los resultados de la clasificación aglomerativa y la prueba de Mantel indican que los cambios florísticos entre las parcelas que se observan en helechos y en los diferentes grupos de árboles son muy semejantes. Este resultado sugiere que cualquiera de estos grupos puede servir como un indicador exacto de los otros grupos. De tal manera, el conocimiento de la composición florística de los helechos, por ejemplo, hace posible predecir con bastante confianza cuales son las especies de árboles que se encuentran en el mismo lugar.

Las agrupaciones de las parcelas obtenidas con las Melastomataceas y las Myristicaceas difieren un poco de las 
obtenidas con helechos y con árboles, y también las correlaciones lineares entre estas familias y los otros grupos son relativamente bajas. A pesar de esto, las correlaciones son estadísticamente significativas, lo cual indica que no son

Tabla 1. Las especies de helechos encontradas en diferentes parcelas.

\begin{tabular}{|c|c|c|c|c|c|c|c|c|c|c|c|c|}
\hline ESPECIES & & & & & & ARC & ;EL & & & & & \\
\hline & 1 & 2 & 3 & 4 & 5 & 6 & 7 & 8 & 9 & 10 & 11 & 12 \\
\hline Adiantum cf. obliquum Willd & & & $x$ & $x$ & & & & & & & & \\
\hline Adiantum terminatum Kunze & & & & & $x$ & $\mathrm{x}$ & $x$ & & & & & \\
\hline Adiantum tetraphyllum Willd & & $x$ & & $\mathrm{x}$ & & & & & & & & \\
\hline Adiantum tomentosum $\mathrm{KI}$. & $\mathrm{x}$ & $x$ & & & $\mathrm{X}$ & $x$ & $x$ & $\mathrm{x}$ & & & & \\
\hline Antrophyum cajenense ( Desv. ) Sprengel & & & $x$ & $\mathrm{x}$ & & & & $\mathrm{X}$ & & & & \\
\hline $\begin{array}{l}\text { Arachniodes macrostegia ( Hooker) Tryon \& } \\
\text { Conant }\end{array}$ & & & & & & & & & $x$ & & & \\
\hline Asplenium escaleroense Chirist & & & & & & & & & & $x$ & & \\
\hline Asplenium hallii Hook. & & & & & $\mathrm{X}$ & $x$ & $x$ & & & & & \\
\hline Asplenium serratum L. & $\mathrm{x}$ & $x$ & $x$ & $\mathrm{x}$ & & & $x$ & $x$ & & & & \\
\hline Campyloneurum phyllitidis ( L ) Presl & $\mathrm{x}$ & & $x$ & $\mathrm{x}$ & & & & & & & & \\
\hline Cyathea bradei (Windish) Lellinger & & & & & & & $\mathrm{x}$ & $\mathrm{X}$ & & & & \\
\hline Cyclodium meniscioides (Willd.) Presl & & $x$ & & & & & $x$ & $\mathrm{x}$ & & & & \\
\hline Danaea elliptica J. E. Smith & $\mathrm{x}$ & $x$ & & & $\mathrm{x}$ & $x$ & $x$ & & & & & \\
\hline Elaphoglossum cf. brachyneuron (Fée ) J. Sm. & & & & & & & & & & & & $x$ \\
\hline Elaphglossum cf. pilosum ( $\mathrm{H}$. B. Willd. ) Moore & & & & & & & & & & & $x$ & \\
\hline Elaphoglossum ct. pteropus C. Chr. & & & & & & & & $x$ & $\mathrm{x}$ & $\mathrm{x}$ & & \\
\hline Elaphoglossum sp. 1 & & $\mathrm{x}$ & & & & & & & & & & \\
\hline Elaphoglossum sp. 2 & $\mathrm{x}$ & & & & & & & & & & & \\
\hline Hecistopteris pumila ( Sprengel ) J. Sm. & & & & & & & $x$ & $x$ & $x$ & $x$ & $x$ & $x$ \\
\hline Lindsaea bolivarensis V. Marcano ( ined. ) & & $\mathrm{x}$ & & & & & & & & & & \\
\hline Lindsea divaricata KI. & & $x$ & $x$ & $\mathrm{x}$ & $x$ & $\mathrm{x}$ & & & $\mathrm{x}$ & $x$ & $x$ & $x$ \\
\hline Lindsea guianensis Dryander & & & & & & & $\mathrm{x}$ & & & & & \\
\hline Lindsaea hemiglossa Kramer & & & & & & & & & & & & $x$ \\
\hline Lindsaea lancea ( L. ) Bedd. & $\mathrm{x}$ & $x$ & & & $x$ & $\mathrm{x}$ & $\mathrm{x}$ & & & & & \\
\hline Lindsaea schomburgkii Klotzch & & & & & & & & & & & $x$ & \\
\hline Lomagramma guianensis ( Aubl. ) Ching & $x$ & & $\mathrm{x}$ & $x$ & & & & & & & & \\
\hline Lomariopsis japurensis (Martius) J. Smith & $x$ & $x$ & $x$ & $x$ & $x$ & $\mathrm{X}$ & $x$ & & & & & \\
\hline Metaxya rostrata ( Willd. ) Presl & & $\mathrm{x}$ & - & & & & $\mathrm{x}$ & $\mathrm{x}$ & & & & \\
\hline Microgramma cf. Ioretense Maxon & $\mathrm{x}$ & $x$ & & & & & & & & $x$ & & $x$ \\
\hline Microgramma lycopodioides ( L. ) Copel. & & $x$ & & & & & $\mathrm{x}$ & & & & & \\
\hline Microgramma sp. 1 & & & & $x$ & & & & & & $=$ & & \\
\hline Nephrolepis rivularis ( Vahl) Mett. ex Krug & & $\mathrm{x}$ & & $\mathrm{X}$ & & & $x$ & $x$ & & & & \\
\hline Polybotrya caudata Kze. & & & $x$ & & & & & & & & & \\
\hline Polybotrya crassirhizoma Lellinger & & & $x$ & & & & & & & & & \\
\hline Polybotrya osmundacea Willd. & $\mathrm{x}$ & & $x$ & $x$ & & & & & & & & \\
\hline Polybotrya pubens Mart. & $\mathrm{x}$ & $\mathrm{X}$ & & & $x$ & $x$ & & & & & & \\
\hline Polypodium s. lat. sp. 1 & & & & $x$ & & & & & & & & \\
\hline Polypodium triseriale Sw. & & $x$ & & & & & & & & & & \\
\hline Pteris altissima Poiret & & & & $\mathrm{x}$ & & & & & & & & \\
\hline Saccoloma elegans Kaulfuss & & & $\mathrm{x}$ & & & & & & & & & \\
\hline Saccoloma inaequale ( Kunze) Mattenius & $\mathrm{x}$ & & $x$ & $x$ & $\mathrm{x}$ & $x$ & $\mathrm{x}$ & & & & & \\
\hline Salpichlaena volubilis ( Kaulf. ) J. Smith & & & $x$ & & & & & & & & & \\
\hline Selaginella flexuosa Spring & & & & & & & & $x$ & & & & \\
\hline Selaginella cf. parkeri ( Hook. \& Grev. ) Spring. & & & & & $\mathrm{x}$ & $x$ & $\mathrm{x}$ & & & & & \\
\hline Thelypteris opulenta ( Kaulf. ) Fosberg & & & $\mathrm{x}$ & $\mathrm{x}$ & & & & & & & & \\
\hline Trichipteris nigra ( Mart.) Tryon & $\mathrm{x}$ & $x$ & $x$ & $\mathrm{x}$ & $x$ & $\mathrm{X}$ & & & & & & \\
\hline Trichomanes ankersii Parker ex Hook. \& Grev. & $\mathrm{X}$ & $\mathrm{x}$ & $x$ & & $x$ & $x$ & $\mathrm{x}$ & $\mathrm{x}$ & $x$ & $x$ & $\mathrm{X}$ & $x$ \\
\hline Trichomanes bicorne Hook. & & & & & & & & $\mathrm{x}$ & $x$ & $x$ & $x$ & \\
\hline Trichomanes cf. crispum $L$. & & $x$ & & & & & & $x$ & $x$ & $x$ & $x$ & \\
\hline Trichomanes elegans Rich. & & & & & $x$ & $\mathrm{x}$ & $\mathrm{x}$ & & & & & \\
\hline Trichomanes martiusii Presl & & & & & & & & $x$ & $x$ & $x$ & $x$ & $\mathrm{x}$ \\
\hline Trichomanes pinnatum hedwig & $x$ & $x$ & & $x$ & $\mathrm{x}$ & $x$ & $x$ & $\mathrm{x}$ & & & & \\
\hline Triplophyllum funestum ( Kze. ) Holtt. & & $x$ & & & $x$ & $x$ & & & & & & \\
\hline
\end{tabular}


Tabla 2. Las especies de Melastomataceae encontradas en diferentes parcelas con sus índices de valor de importancia.

\begin{tabular}{|c|c|c|c|c|c|c|c|c|c|c|c|c|}
\hline \multirow[t]{2}{*}{ ESPECIE } & \multicolumn{12}{|c|}{ PARCELA } \\
\hline & 1 & 2 & 3 & 4 & 5 & 6 & 7 & 8 & 9 & 10 & 11 & 12 \\
\hline $\begin{array}{l}\text { Adelobotrys cf. adscendens ( Sw. ) } \\
\text { Tr. }\end{array}$ & & 14 & & & & & & & & & & \\
\hline Adelobotrys cf. boissierina Cogn. & 9 & & & & 6 & 15 & 20 & & & & & \\
\hline $\begin{array}{l}\text { Adelobotrys intonsa (Gleason) } \\
\text { Wurdack }\end{array}$ & & & & & 44 & 25 & 18 & & & & & \\
\hline Clidemia epibaterium DC. & & & & & & & & & & 105 & 48 & 22 \\
\hline Clidemia epiphytica (Tr.) Cogn. & & & & $2:$ & 27 & 45 & 13 & 127 & & & & \\
\hline $\begin{array}{l}\text { Leandra candelabrum (Macbr.) } \\
\text { Wurdack }\end{array}$ & 27 & 35 & & & & & & & & & & \\
\hline Leandra secunda (D. Don) Cogn. & & & & & & 9 & & & & & & \\
\hline $\begin{array}{l}\text { Loreya umbellata ( Gleason) } \\
\text { Wurdack }\end{array}$ & & & & 20 & 112 & 74 & 130 & 33 & & & & \\
\hline Maieta guianensis Aubl. & & & & & & & & 5 & & & & \\
\hline $\begin{array}{l}\text { Miconia barbinenvis ( Benth) } \\
\text { Triana }\end{array}$ & & & & & 11 & 30 & 15 & 4 & & & & \\
\hline Miconia carassana Cogn. & & 10 & & & & & & & & & & \\
\hline Miconia dolichorryncha Naud. & & & & & 3 & 16 & & 4 & & & & 12 \\
\hline Miconia cf. dorsiloba Gleason & 81 & & & & & & & & & & & \\
\hline Miconia lamprophylla Triana & & & 34 & & & & & & & & & \\
\hline Miconia nervosa ( Smith) Tr. & & & & 64 & & & & & & & & \\
\hline Miconia cf. pilgeriana Ule & & & & & 15 & 9 & & & & & & \\
\hline Miconia pterocaulon $\mathrm{Tr}$. & & & & & & & & 26 & & & & \\
\hline Miconia cf. punctata ( Desr.) Don & 25 & 88 & & & 24 & 13 & 19 & 5 & & & & 19 \\
\hline $\begin{array}{l}\text { Miconia radulaefonia (Benth.) } \\
\text { Naud. }\end{array}$ & & & & & & 3 & & & & & & \\
\hline Miconia rugosa $\mathrm{Tr}$. & & & & & & & 16 & & & & & \\
\hline $\begin{array}{l}\text { Miconia tomentosa (I.C.Rich. ) } \\
\text { DON }\end{array}$ & 7 & & & 28 & 3 & & & & & & & \\
\hline Miconia traillii Cogn. & 22 & & 49 & 59 & & & & & & & & \\
\hline Miconia sp. 1 & & & & & & & & 4 & & & & \\
\hline Miconia sp. 2 & & & & 21 & & & & & & & & \\
\hline Miconia sp. 3 & 10 & & & & & & & & & & & \\
\hline Ossaea araneifera Mkgf. & & & & & 27 & 11 & 22 & & & & & \\
\hline Ossaea cucullata Gleason & & & & & 16 & 3 & 7 & & & & & \\
\hline Tococa guianensis Aubl. & 119 & 153 & 217 & 82 & & 6 & 36 & 79 & 300 & 195 & 252 & 247 \\
\hline Tococa cf. ulei Pilger & & & & & 12 & 41 & 4 & 13 & & & & \\
\hline Suma & 300 & 300 & 300 & 300 & 300 & 300 & 300 & 300 & 300 & 300 & 300 & 300 \\
\hline
\end{tabular}


Tabla 3. Las especies de Myristicaceae encontradas en diferentes parcelas con sus índices de valor de importancia.

\begin{tabular}{|c|c|c|c|c|c|c|c|c|c|c|c|c|}
\hline ESPÉCIE & & & & & & PARC & SELA & & & & & \\
\hline & 1 & 2 & 3 & 4 & 5 & 6 & 7 & 8 & 9 & 10 & 11 & 12 \\
\hline $\begin{array}{l}\text { Compsoneura capitellata } \\
\text { (D C. ) Ward }\end{array}$ & 17 & 32 & & & & & & & & & & \\
\hline Iryanthera elliptica Duck & 62 & & & & 28 & & 15 & & & & & \\
\hline $\begin{array}{l}\text { Iryanthera cf. juruensis } \\
\text { Warb. }\end{array}$ & & & 101 & 20 & & & 14 & 28 & & & & \\
\hline Iryanthera lancifolia Duck & & & & & & 40 & 16 & 24 & & & & \\
\hline $\begin{array}{l}\text { Iryanthera macrophylla } \\
\text { (Benth. ) Warb. }\end{array}$ & 28 & 33 & 10 & 42 & & 31 & & 21 & & & & \\
\hline Iryanthera cf. paraensis Hub. & 15 & 30 & & 96 & & 38 & & 116 & & & & \\
\hline Iryanthera polyneura Duck & & & & & 43 & 94 & & & & & 49 & 93 \\
\hline Iryanthera tricornis Ducke & & 67 & & & 82 & 47 & 121 & & & & & \\
\hline Iryanthera ulei Warb. & & & & & 12 & & & 48 & & 82 & 126 & 119 \\
\hline $\begin{array}{l}\text { Osteophloem platyspermum } \\
\text { (DC.) Warb. }\end{array}$ & & & & & 17 & & 14 & & & & & \\
\hline $\begin{array}{l}\text { Otoba glycycarpa (Ducke) } \\
\text { Rodr. }\end{array}$ & & & 25 & & & & & & & & & \\
\hline Virola calophylla Warb. & 36 & & 61 & 72 & 62 & 16 & 33 & 42 & & & 37 & \\
\hline Virola divergens Duck & & & & & 8 & & & & & & & \\
\hline $\begin{array}{l}\text { Virola elongata ( Benth. ) } \\
\text { Warb. }\end{array}$ & & & & & 27 & 17 & 45 & & & & & \\
\hline $\begin{array}{l}\text { Virola cf. flexuosa A. C. } \\
\text { Smith }\end{array}$ & & & 12 & & & & & & & & & \\
\hline Virola marlenei Rodr. & & & & & 8 & 8 & 28 & & & & & \\
\hline Virola multinervia Duck & 38 & & & & & & & & & & & \\
\hline Virola obovata Duck & 41 & 9 & & & & & 14 & & & & & \\
\hline Virola pavonis ( DC. ) Smith & 32 & 129 & 81 & 50 & 13 & 9 & & 21 & 300 & 218 & 88 & 88 \\
\hline $\begin{array}{l}\text { Virola peruviana ( DC. ) } \\
\text { Warb }\end{array}$ & & & & 20 & & & & & & & & \\
\hline Virola sp. 1 & 17 & & & & & & & & & & & \\
\hline Virola sp. 2 & 14 & & 10 & & & & & & & & & \\
\hline Suma & 300 & 300 & 300 & 300 & 300 & 300 & 300 & 300 & 300 & 300 & 300 & 300 \\
\hline
\end{tabular}


Tabla 4. Indice de valor de importancia de cada familia (IVIF) en cada parcela. El IVIF está calculada según la manera presentada por CURTIS \& MCINTOSH (1950), en que IVIF = diversidad relativa + densidad relativa + dominancia relativa.

\begin{tabular}{|c|c|c|c|c|c|c|c|c|c|c|c|c|}
\hline FAMÍLIA & & & & & & PARC & SELA & & & & & \\
\hline & 1 & 2 & 3 & 4 & 5 & 6 & 7 & 8 & 9 & 10 & 11 & 12 \\
\hline Anacardiaceae & 41,1 & 3,7 & & & 1,8 & & 7,9 & & 1,5 & & & 1,6 \\
\hline Annonaceae & 11,0 & 20,5 & 14,7 & 11,1 & 7,7 & 12,0 & 11,9 & 16,6 & 13,4 & 17,1 & 10,1 & 15,2 \\
\hline Apocynaceae & 1,8 & 1,8 & 1,7 & 3,7 & 1,6 & & 8,5 & 7,9 & 10,0 & 13,8 & 10,4 & 8,4 \\
\hline Aquifoliaceae & & & & & & & & & 1,4 & & 1,9 & \\
\hline Araliaceae & 3.6 & & 5,6 & & & & 1,7 & 2,0 & 1,4 & 3,1 & & \\
\hline Arecaceae & 7,5 & 2,0 & 2,5 & 3,3 & 23,4 & 29,4 & 3,1 & 26,2 & & & & \\
\hline Bignoniaceae & & & & & 8,0 & 3,0 & & 3,0 & 6,0 & 3,2 & 1,9 & 1,5 \\
\hline Bombacaceae & & & 4,1 & & & & & & 7,0 & 10,6 & & \\
\hline Boraginaceae & 1,8 & & 3,4 & & 3,4 & 1,5 & & 6,1 & & & & \\
\hline Burseraceae & 20,6 & 22,7 & 36,2 & 16,7 & 14,4 & 10,7 & 19,8 & 5,2 & 4,3 & & & 3,1 \\
\hline Caryocaraceae & 1,8 & & & 9,7 & & & & & & & & \\
\hline Celastraceae & 2,0 & & & & & & & & & & & \\
\hline Chrysobalanaceae & 5,2 & 24,9 & 9,3 & 10,0 & 8,0 & 6,9 & 21,0 & 9,9 & 1,6 & 3,6 & 1,9 & 9,1 \\
\hline Combretaceae & 2,0 & 1,8 & & 7,2 & & & & 5,2 & 1,4 & & 2,5 & \\
\hline Connaraceae & 1,8 & & & & & & & & & & & \\
\hline Dichapetalaceae & & & & 1,5 & & & & & & & & \\
\hline Ebenaceae & & & & & & & & 2,6 & 1,3 & & & 3,1 \\
\hline Elaeocarpaceae & 14,7 & 8,1 & & 2,7 & 1,6 & & 7,2 & 1,7 & & & 2,0 & \\
\hline Erythroxylaceae & & & & & 1,6 & & & & & & & \\
\hline Euphorbiaceae & 3,6 & 10,7 & 8,4 & 9,6 & 11,8 & 16,8 & 7,6 & 16,0 & 23,1 & 7,9 & 17,0 & 12,0 \\
\hline Flacourtiaceae & 1,8 & 1,8 & 16,4 & 19,4 & & 1,6 & & 7,4 & 4,0 & 7,0 & & 6,5 \\
\hline Guttiferae & 1,8 & 3,6 & 4,3 & 1,4 & 6,0 & 10,8 & 7,8 & 34,9 & 6,5 & 35,2 & 14,7 & 7,0 \\
\hline Hippocrateaceae & & 1,8 & & & & & & & & & & \\
\hline Humiriaceae & & 2,0 & & & & & 5,6 & & 1,4 & 1,4 & & \\
\hline Icacinaceae & 2,5 & & & & & & 10,9 & & 4,6 & 1,4 & & 3,6 \\
\hline Lacistemataceae & 1,8 & 1,8 & & & & & & & & & & \\
\hline Lauraceae & 22,2 & 27,6 & 46,0 & 17,4 & 8,1 & 21,3 & 26,1 & 22,7 & 13,4 & 2,9 & 2,0 & 12,1 \\
\hline Lecythidaceae & 9,8 & 25,3 & 3,4 & 13,5 & 27,4 & 24,3 & 8,0 & & & & & \\
\hline Leguminosae & 5,6 & 9,8 & 27,5 & 34,4 & 15,0 & 26,4 & 21,3 & 28,3 & 38,8 & 57,0 & 70,1 & 44,7 \\
\hline Linaceae & 1,8 & & & & & & & & 1,3 & 6,3 & & \\
\hline Loganiaceae & & & & & & & & & 1,3 & 4,0 & 1,9 & 3,7 \\
\hline Magnoliaceae & & & 2,4 & & & & & 1,8 & & & & \\
\hline Malpighiaceae & 5,0 & & & & & & 6,3 & & 5,3 & & & 4,9 \\
\hline Melastomataceae & 9,0 & 5,9 & 5,8 & 4,2 & 4,9 & 7,3 & 5,4 & 5,1 & & & & 1,5 \\
\hline
\end{tabular}


continuación (Tabla 4)

\begin{tabular}{|c|c|c|c|c|c|c|c|c|c|c|c|c|}
\hline \multirow[t]{2}{*}{ FAMÍLIA } & \multicolumn{12}{|c|}{ PARCELA } \\
\hline & 1 & 2 & 3 & 4 & 5 & 6 & 7 & 8 & 9 & 10 & 11 & 12 \\
\hline Meliaceae & 3,7 & 7,9 & 3,4 & 15,8 & 19,9 & 12,9 & 15,6 & 5,0 & 4,1 & 3,1 & 3,7 & 3,1 \\
\hline Monimiaceae & 7,3 & 3,6 & 8,8 & 8,1 & & 3,0 & & 3,8 & & & & \\
\hline Moraceae & 19,2 & 37,6 & 8,4 & 12,5 & 13,8 & 27,0 & 7,0 & 17,9 & 2,7 & & 1,9 & 4,5 \\
\hline Myristicaceae & 35,5 & 27,8 & 30,5 & 14,0 & 35,5 & 34,1 & 30,5 & 18,2 & 2,8 & 5,5 & 16,0 & 9,2 \\
\hline Myrsinaceae & & & & & & & 3,3 & & 4,0 & 5,4 & & 7,5 \\
\hline Myrtaceae & 2,1 & 3,5 & 1,8 & 13,8 & 1,6 & 1,5 & 1,6 & 8,2 & 7,6 & 7,4 & 7,7 & 14,9 \\
\hline Nyctaginaceae & & & 4,1 & 9,8 & & & & 1,9 & 13,3 & 4,8 & 11,5 & 11,8 \\
\hline Ochnaceae & & & & & & & & 3,4 & & 1,5 & & \\
\hline Olacaceae & & & 9,9 & 1,4 & 5,6 & 1,7 & 5,7 & 4,3 & 6,3 & 7,7 & 3,7 & 2,9 \\
\hline Oleaceae & & & & & & & & & 3,3 & & & \\
\hline Piperaceae & 4,5 & & 1,7 & 4,6 & & & & & & & & \\
\hline Proteaceae & 8,8 & & & & & & & & & & & \\
\hline Quiinaceae & 1,8 & & & & & & & & & & & \\
\hline Rhizophoraceae & & & & & 2,3 & & 5,5 & & & & & \\
\hline Rubiaceae & 10,8 & 19,2 & 15,3 & 9,9 & 6,4 & 6,7 & 1,7 & 8,7 & 9,1 & 10,7 & 8,6 & 8,7 \\
\hline Rutaceae & & & 2,5 & 7,4 & & & & & 10,5 & 13,1 & 28,9 & 1,5 \\
\hline Sabiaceae & & & 3,9 & 1,7 & 1,8 & 9,2 & 1,5 & 4,4 & 1,4 & & & \\
\hline Sapindaceae & & 1,9 & & & 2,6 & & 3,4 & 11,9 & 7,7 & 6,9 & 5,1 & 48,7 \\
\hline Sapotaceae & 8,2 & 9,6 & 7,0 & 19,2 & 31,0 & 13,1 & 20,1 & 6,3 & 45,3 & 30,9 & 66,9 & 31,8 \\
\hline Simaroubaceae & 6,0 & & & & & & & & & & & \\
\hline Sterculiaceae & & 5,4 & 3,7 & 6,8 & 1,6 & 6,7 & 3,4 & 3,4 & & & & \\
\hline Theaceae & & & & & & & & & & 6,3 & 3,6 & 3,7 \\
\hline Tiliaceae & & & & & & 1,5 & & & & & & \\
\hline Ulmaceae & & & 1,9 & 1,7 & & & & & & & & \\
\hline Violaceae & 7,5 & 7,7 & 1,9 & 7,5 & 8,8 & 1,5 & 14,5 & & & & & \\
\hline Vochysiaceae & & & & & & & 2,2 & & & & & \\
\hline Familia desconoc. & & & & & & & & & 4,4 & & 1,1 & 1,2 \\
\hline $\begin{array}{l}\text { Muestra perdida o } \\
\text { confundida }\end{array}$ & 4,8 & & 3,5 & & 24,4 & 9,1 & 3,9 & & 28,5 & 22,2 & 4,9 & 12,5 \\
\hline Suma & 300 & 300 & 300 & 300 & 300 & 300 & 300 & 300 & 300 & 300 & 300 & 300 \\
\hline
\end{tabular}

resultados de procesos aleatorios. Aparentemente, las distribuciones de las especies de estas dos familias siguen patrones semejantes a los encontrados en las otras especies estudiadas, aunque es posible que las correlaciones más bajas indiquen algunas diferencias ecológicas. También es posible que las correlaciones bajas resulten de variación aleatoria: las Melastomataceas y las Myristicaceas presentan menos especies e individuos que los otros grupos en el presente estudio. Nuestros datos no permiten evaluar cual de estas razones 
explica mejor las diferencias.

La potencialidad práctica de usar algún grupo de plantas como un indicador de los otros componentes de la flora depende de la semejanza de sus comportamientos, pero también de la cantidad de trabajo que se necesita para revelar los patrones en su distribución. Por ejemplo, en el presente estudio, practicalmente, los mismos resultados fueron conseguidos utilizando árboles grandes, árboles
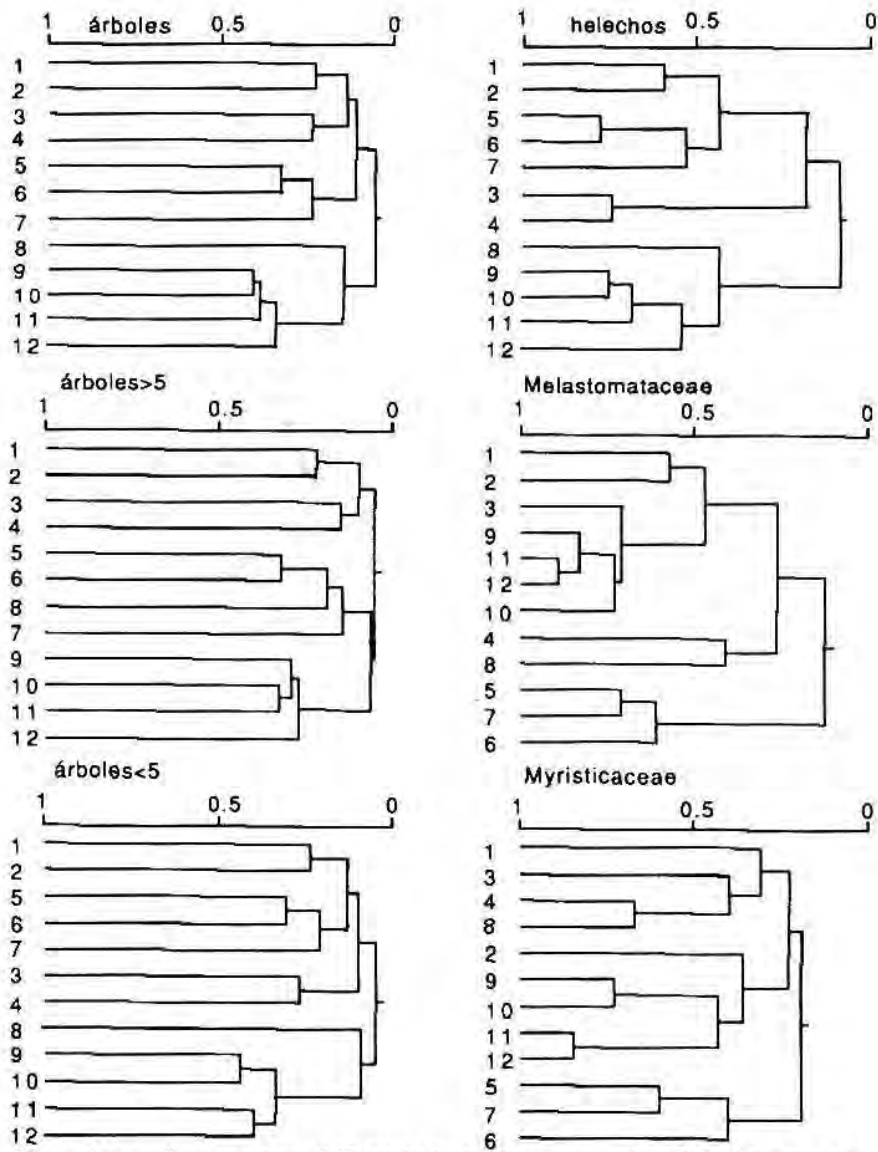

Figura 2. Classificación aglomorativa (UPGMA) de doce parcelas 0.0625 ha cada una basada en el porcentaje de similitud (PS) o en el índice de JACCARD (J) según a) árboles mayores de $2.5 \mathrm{~cm}$ DAP (PS), b) árboles mayores de $5 \mathrm{~cm}$ DAP (PS), c) árboles entre $2.5 \mathrm{~cm}$ y $5 \mathrm{~cm} \mathrm{DAP}$ (PS), d) helechos (J), e) Melastomataceae (PS), y f) Myristicaceae (PS). 
Tabla 5. Correlación estandardizada entre diferentes grupos taxonómicos y fisonómicos según la prueba de Mantel. Las probabilidades (P) de observar correlaciones iguales o mayores al azar están indicadas por estrellas: $* *=\mathrm{P}<0.01, * * *=\mathrm{P}<0.001$

\begin{tabular}{|c|c|c|c|c|c|}
\hline & Melastomataceae & Myristicaceae & $\begin{array}{l}\text { Todos los } \\
\text { árboles }\end{array}$ & $\begin{array}{l}\text { Árboles } \\
\text { mayores de } \\
5 \mathrm{~cm} \text { DAP }\end{array}$ & $\begin{array}{l}\text { Arboles } \\
\text { menores de } \\
5 \mathrm{~cm} \text { DAP }\end{array}$ \\
\hline Helechos & $0.396^{\star \star}$ & $0.531^{* * *}$ & $0.823^{\star \star \star}$ & $0.783^{\star \star *}$ & $0.820^{\star \star *}$ \\
\hline Melastomataceae & & $0.614^{\star \star \star}$ & $0.553^{\star \star \star}$ & $0.548^{\star \star \star}$ & $0.549^{* \star *}$ \\
\hline Myristicaceae & & & $0.582^{\star \star}$ & $0.555^{* *}$ & $0.627^{\text {***}}$ \\
\hline $\begin{array}{l}\text { Arboles mayores } \\
\text { de } 5 \mathrm{~cm} \text { DAP }\end{array}$ & & & & & 0.902 \\
\hline
\end{tabular}

mente, para la selección del grupo indicadorio hay que hallar una combinación óptima entre la escala geográfica del estudio, la mano de obra disponible y la exactidud requerida en el estudio.

Los resultados del presente estudio sugieren la existencia de verdaderas comunidades vegetales en los bosques pluviales de la Amazonia. La buena correlación fitosociológica entre los taxonómicamente y estructuralmente diferentes grupos de plantas indica que las especies no están distribuidas al azar. Esta observación limita significativamente la aplicabilidad de varios modelos que suponen que la composición florística de los bosques pluviales es el resultado de algún proceso aleatorio (CONNELL, 1978; DAVIS, 1986; HUBBELL \& FOSTER, 1986). En vez de ello, parece que existe uno o varios factores que determinan de manera previsible la estructura florística de los bosques. Tales factores pueden ser por ejemplo el contenido de nutrientes en el suelo (TILMAN,
1986), las condiciones de drenaje (KAHN \& CASTRO, 1985; LIEBERMAN et al., 1985; POUSEN \& BALSLEV; 1991), el estadio sucesional (GRUBB, 1977; DENSLOW, 1987) etc. En el presente estudio las diferencias en la geomorfología están correlacionadas con las características florísticas observadas. En la mayoría de las clasificaciones las parcelas en la parte colinosa y en la zona baja de Mishana, asi como las parcelas de la parte ondulada y las de la terraza baja en Momón se separaron en grupos propios. También las características particulares de la parcela 8 situada en la zona transicional entre la terraza baja y el terreno colinoso en Mishana confirman esta idea. Aunque el análisis de los suelos está incompleto todavía, parece que en el área de estudio el tipo del suelo también está correlacionado con la geomorfología. La parte colinosa en Mishana presenta arena blanca, que es un sustrato conocido por su baja fertilidad (ANDERSON, 1981). Las terrazas de los ríos Momón y Nanay probablemente tienen suelos 
diferentes porque los ríos son químicamente diferentes (KALLIOLA et al., 1993). En la parte colinosa en Momón la temperización y erosión han afectado el suelo por mucho más tiempo que en la terraza baja del río Momón, y por eso se puede esperar que las caracteristicas del suelo son diferentes.

Los resultados aquí expuestos apoyan la idéa de equilibrium en la composición florística de los bosques pluviales, y además proponen una altemativa relativamente rápida y fácil para la clasificación de la vegetación y los estudios fitogeográficos de la Amazonía. Sin embargo, hay que mantener ciertas reservas. El presente estudio incluye sólo un área relativamente pequeña y con pocas parcelas. Además, cuatro de las parcelas representan áreas con arena blanca, que se conocen muy distintas de otros ambientes en la tierra firme peruana. Por eso, los resultados no pueden ser generalizados a otras regiones antes de haber realizado estudios más amplios en el campo.

\section{AGRADECIMIENTOS}

\section{Agradecemos Juan Ruiz Macedo} por su indispensable ayuda en varias fases del trabajo, y Proyecto Flora del Perú del Missouri Botanical Garden ofreció algunos instrumentos del campo y un excelente secador de muestras botánicas para nuestro uso. Tania de la Rosa colaboró en la revisión y el lenguaje del manuscrito. Comelius C. Berg, Al Gentry, Bruce Holst, Ron Liesner, Paul Maas, Robbin C. Moran, Mónica Palacios Ríos, Terry Pennington, Susanne S. Renner, Carlos Reynell y Charlotte Taylor han ayudado en la identificación de las muestras botánicas, y
Pierre Legendre y Daniel Borcard en los análisis estadísticos. También agradecemos la colaboración de los habitantes del pueblo de Mishana y del personal de Amazon Selva Tours durante el trabajo del campo. Risto Kalliola, Maarit Puhakka y Rodolfo Vásquez han dado comentarios valorables para mejorar el manuscrito. El estudio fue financiado por FINNIDA y la Academia de Finlandia.

\section{Bibliografia citada}

ANDERSON, A. B. - 1981. White-sand vegetation of Brazilian Amazonia. Biotropica, 13(3):199-210.

ASHTON, P. S. - 1969. Speciation among tropical forest trees: some deductions in the light of recent evidence. Biol. J. Linn. Soc, 1:155196.

BRAUN-BLANQUET, J. - 1932. Plant sociology: The study of plant communities. Londres. Hafner. 439 p.

BRAY, J. R. \& CURTIS, J. T. - 1957. An ordination of the upland forest communities of southern Wisconsin. Ecological Monographs, 27:325-349.

CAJANDER, A. K. - 1909. Über Waldtypen. Acta Forest. Fennica, 1:1-175.

CAMPBELL, D. G.; DALY, D. C.; PRANCE, G. T.; MACIEL, U. N. - 1986. Quantitative ecological inventory of terra firme and várzea tropical forest on the Río Xingu, Brazilian Amazon. Brittonia, 38:369-393.

CAMPBELL, D. G. \& HAMMOND, H. D. (eds.) - 1989. Floristic lnventory of Tropical Countries. Nueva York. New York Botanical Garden.

CONNEL, J. H. - 1978. Diversity in tropical rain forests and coral reefs. Science, 199:13021310.

CONSERVATION INTERNATIONAL - 1991.

Prioridades Biológicas para la Consenvaciónde la Amazonia. La mapa final del taller "Workshop 90 - Prioridades Biológicas para la Conservación de la Amazonici". Washington. IBAMA, INPA y 
Conservation International.

CURTIS, J. T. \& MCLNTOSH, R. P. - 1950. The interrelation of certain analytic andsynthetic phytosociological characters. Ecology, 31:435-455.

DAVIS, M. B. - 1986: Climatic instability, time lags, and community disequilibrium. En: Community Ecology. Diamond, J. \& Case, T. J. (eds.). Nueva York. Harper and Row. p. $269-284$.

DENSLOW, J. S. - 1987. Tropical rainforest gaps and tree species diversity. Ann. Rev. Ecol. Syst., 18:431-451.

ENCARNACIÔN, F - 1985. Introducción a la flora y vegetación de la Amazonia peruana: estado actual de los estudios, medio natural y ensayo de una clave de determinación de las formaciones vegetales en la llanura amazónica. Candollea, 40:237-252.

GENTRY, A. H. - 1981. Distributrional pattems and an additional species of the Passiflora vitifolia complex: Amazonian species diversity due to edaphically differentiated communities. PI. Syst. Evol., 137: 95-105.

1988. Changes in plant community diversity and floristic composition on environmental and geographic gradients. Ann. Missouri Bot. Gard., 75:1-34.

GRUBB, P. J. - 1977. The maintenance of speciesrichness in plant communities: The importance of the regeneration niche. Biol. Rev., 52:107-145.

HAFFER, J. - 1969. Speciation in Amazonian forest birds. Science, 165:131-137.

HUBBELL, S. P. \& FOSTER, R. B. - 1986. Biology, chance, and history and the structure of tropical rain forest tree communities. En: Community ecology. Diamond, J. \& Case, T. J. (eds.). Nueva York. Harper \& Row Publishers. p. 314-329.

JACCARD P. - 1901. Distribution de la flore alpine dans le Bassin des Dranses et dans quelques régions voisines. Bull. Soc, vand. Sci. Nat., 37: 24I-272.

KAHN, F. \& CASTRO, A. - 1985. The palm community in a forest of central Amazonia, Brazil. Biotropica, 17(3): 210-216.

KALLIOLA, R.; LINNA, A.; PUHAKKA, M.;
SALO, J.; RASANEN, M. - 1993, Mineral nutrients in river sediments from Peruvian Amazonia: provenance and vegetational implications. Catena, 20(3):333-349.

LEGENDRE, P. \& VAUDOR, A. - 1991. The $R$ package: Multidimensional analysis, spatial analysis. Département de sciences biologiques, Université de Montréal. iv + $142 \mathrm{p}$.

LIEBERMAM, M.; LIEBERMAM, D.; HARTSHORN, G. S.; PERALTA, R. 1985. Small-scale altitudinal variation in lowland wet tropical forest vegetation. $J$. Ecol, 73:505-516.

MANTEL, N. - 1967. The Detection of Disease Clustering and a Generalized Regression Approach. Cancer Research, 27(2):209-220.

NELSON, B. W.; FERREIRA, C. A.; da SILVA, M.F; KAWASAKI, M. L. - 1990. Refugia, endemism centers and botanical collecting density in Brazilian Amazonia. Nature, 345:714-716.

PEÑAHERRERA, del A. C - 1986. Geografía Fisica del Perú. En: Gran Geografia del Perú, Naturaleza y Hombre. Manfer - Mejia Baca, J. (ed.), Barcelona. Vol. 1.

POULSEN, A. D. \& BALSLEV, H. - 1991. Abundance and cover of ground herbs in an Amazonian rain forest. Journal of Vegetation. Science, 2: 315-322.

PRANCE, G. T. - 1973. Phytogeographic support for the theory of Pleistocene refuges in the Amazon Basin, based on evidence from distribution patterns in Caryocaraceae, Chrysobalanaceae, Dichapetalaceae and Lecythidaceae. Acta Amazonica, 3(3): 5-28.

1982. Forest refuges: evidence from woody angiosperms. En: Biological diversification in the tropics. Prance, G. T. (ed.). Nueva York. Columbia University Press. p. 137 157.

RUOKOLAINEN, K. \& TUOMISTO, H. - 1993. La vegetación de terrenos no inundables (tierra firme) en la selva baja de la Amazonia Peruana. En: Amazonia Peruana vegetación húmeda tropical en el llano subandino. Kalliola, R.; Puhakka, M.; Danjoy, W. (eds.). Jyväskylä. PAUT y ONERN. p. 139-153. 
SALOMÃO, R. P.; SILVA M. F. F.; ROSA, N. A. - 1988. Inventário ecológico em floresta pluvial tropical de terra firme, Serra Norte, Carajás, Pará. Bol. Mus. Para. Emílio Goeldi, Sér. Bot., 4(1):1-46.

SMOUSE, P. E.; LONG, J. C.; SOKAL, R. R. 1986. Multiple Regression and Correlation Extensions of the Mantel Test of Matrix Correspondence. Syst. Zool., 35(4):627-632.

SOKAL, R. R. \& MICHENER, C. D. - 1958. A Statistical Method for Evaluating Systematic Relationships. Univ. Kansas Sci. Bull., 38(22): 1409-1438.

TILMAN, D. - 1986. Resources, competition and the dynamics of plant communities. En: Plant ecology. M. J. Crawley (ed.). Oxford. Blackwell Scientific Publications. p. 51-75.
TUOMISTO, H.; LINNA, A.; KALLIOLA, R. (en prensa), Use of digitally processed satellite images in studies of tropical rain forest vegetation. International Journal of Remote Sensing.

TIOMISTO, H. \& RUOKOLAINEN, K. - (en prensa). Distribution of Pteridophyta and Melastomataceae across an edaphic gradient in non-flooded rain forest in Peruvian Amazonia. Journal of Vegetation Science.

WEBB L. J.; TRACEY, W. T.; WILLIAMS, W. T.; LANCE, G. N. - 1967. Studies in the numerical analysis of complex rain-forest communities II. The problem of species-sampling. J. Ecol, 55: 525-538.

WHITTAKER, R. H. - 1962. Classification of natural communities. Botan. Rev., 28; 1-239. 